\title{
¿LA SOCIEDAD POST-CONFLICTO (LA PAZ DEL CEMENTERIO) O LA GESTIÓN SOCIAL DEL CONFLICTO MEDIANTE LA PARTICIPACIÓN DEMOCRÁTICA?: UNA MIRADA PSICOSOCIAL DEL CONFLICTO ARMADO EN COLOMBIA
}

\section{THE POST-CONFLICT SOCIETY (THE PEACE OF CEMETERY) OR THE SOCIAL MANAGEMENT OF CONFLICT THROUGH DEMOCRATIC PARTICIPATION? : A PSYCHOSOCIAL VIEW OF THE ARMED CONFLICT IN COLOMBIA}

\author{
Miguel Ángel Miranda Rodríguez ${ }^{1}$ \\ Universidad Nacional Abierta y a Distancia, UNAD
}

\section{Resumen}

La sociedad post-conflicto que se vaticina en el discurso gubernamental a propósito de los diálogos en La Habana (Cuba), invita a pensar en las representaciones que se hacen de la sociedad, en el entendido que dichas representaciones están en el origen del conflicto que se pretende dirimir. El documento explora las alternativas a este respecto, descubriendo el anacronismo de las posturas estatales, la invisibilidad de la propuesta guerrillera y la ausencia de visiones alternas, que deberían hacer parte de la discusión. Al final se concluye con la necesidad de oxigenar el proceso, mediante la admisión de temas que convoquen la diversidad de intereses ciudadanos y tiendan a generar un ambiente de confianza, sobre la seguridad proveniente de prestaciones sociales estabilizadas.

Palabras clave: conflicto, sociedad, psicosocial, participación, democracia, gestión social, riesgo, confianza.

\section{Abstract}

The post-conflict society that is predicted in the government discourse at Havana (Cuba) dialogues, invites society to think about the representations that are made of society, on the understanding that such representations are at the root of the conflict that it is intended to resolve. The document explores alternatives in this regard, revealing the anachronism of state positions, the invisibility of a guerrilla proposal and the absence of alternative views, which should be part of the discussion. In the end it concludes with the need to oxygenate the process, by the admission of issues to convene diverse interests and citizens and tend to create an atmosphere of confidence, assured on social security benefits from stabilization.

Keywords: conflict, society, psychosocial, participation, democracy, social management, risk, trust.

Recibido: 15 de julio de 2015

Aceptado: 11 de septiembre de 2015

1 Tutor Tiempo Completo UNAD, CEAD Barranquilla, Colombia, Psicólogo de la Universidad Nacional, Especialista en Clínica y Magister en Psicología de la Universidad del Norte. Correo: miguel.miranda@unad.edu.co. 


\section{Introducción}

Se dice de los diálogos en La Habana entre el gobierno Colombiano y la guerrilla de las FARC, que han polarizado la opinión y exacerbado el unanimismo ideológico, al punto de señalar como preferente la distinción entre amigos y enemigos del proceso. En aras de contribuir con visiones alternas, que por un lado disminuyan expectativas de una sociedad sin conflictos irrealizable, pero que por otro lado aumenten las expectativas de una mayor participación democrática en nuestra convivencia cotidiana; en este documento se propone un análisis de representaciones alternas de la sociedad, con sus correspondientes implicaciones para su actual reproducción. Se contraponen principalmente la representación de una sociedad estratificada, frente a la operación de una sociedad funcionalmente organizada, que ya no se funda en la autoridad personal de dirigentes ilustrados, sino que se anima por el interés de sus ciudadanos para implicarse en la actividad social con sentidos diversos.

\section{La utopía de la sociedad post-conflicto: la paz del cementerio}

Una referencia semántica común, en los actuales diálogos de La Habana entre el Gobierno Colombiano y la guerrilla de las FARC, es la idea de sociedad. Y se podría decir que el conflicto armado entre el gobierno y la guerrilla se justifica desde cada lado, por el tipo de sociedad que defienden, a futuro. Esta sociedad utópica es el referente que integra y compone, en el ámbito de lo simbólico, a los bandos en conflicto. El contenido específico de esta sociedad se da por descontado (es natural) del lado del gobierno y del lado de la guerrilla, se postula como promesa revolucionaria. De ahí que, en lo social, el gobierno descalifique como subversiva la acción de la guerrilla y la guerrilla justifique su accionar bélico, por la intransigencia estatal. Estas relaciones mutuamente constituyentes, entre lo semántico (simbólico) y lo pragmático (político) del discurso de La Habana (Schmidt, 1999), requiere contextualizarse para el estamento civil, con la mediación de la tradición académica (Luhmann, Complejidad y Modernidad. De la Unidad a la Diferencia, 1998), para que los contenidos de sociedad que se esgrimen como justificación de la guerra, publicitados por los medios de comunicación como finalidad de los diálogos en La Habana (la terminación del conflicto), y que promueven la expectativa de una consiguiente pacificación de la nación, con los correspondientes beneficios de prosperidad económica y seguridad social; sean complementados con las acciones civiles necesarias, para que la reproducción cotidiana de lo social, sustituya el recurso a las armas, por la gestión civilizada de la participación democrática (Arendt, 1993).

Las expectativas resultantes de la publicidad de los diálogos en La Habana (Luhmann, The Reality of the Mass Media, 1996), están generando ánimos polarizados mediante la distinción entre amigos y enemigos de la paz. Por supuesto, la simplicidad de tal distinción, distrae de la complejidad de otros temas implicados en el proceso de construcción de la convivencia: 1) el reconocimiento y la administración de los recursos naturales de la nación (no sólo los que son de inmediata explotación económica como el petróleo, el carbón y el oro), sino la tierra, el ciberespacio, los mares y los ríos; 2) el reconocimiento y la gestión de la diversidad cultural de los habitantes de las distintas regiones de la nación, oculta tras la distinción amigos / enemigos; 3) la eficiencia y eficacia del Estado en la garantía de los derechos constitucionales a la salud, la educación y el trabajo digno; y 4) la participación democrática en el gobierno de la nación. De ahí que una aclaración de los proyectos en conflicto, que no sólo incluya la dimensión semántica de sociedad, sino también los aspectos pragmáticos de su permanente reproducción, pueda contribuir a que las expectativas ciudadanas estén más cerca de sus posibilidades de actuación (empoderamiento) (Rey, 2000). 


\section{La sociedad según el establecimiento}

En el discurso gubernamental, pueden distinguirse, además de la faceta política, la jurídica, la económica y la social. El discurso político apela retóricamente a cada una de las otras facetas en el contexto de un esfuerzo "generoso" de conciliación, cuya finalidad es alcanzar la prosperidad prometida durante la campaña electoral. En esa tarea, el discurso jurídico emerge como condicionante inamovible, dado que se remite a la legislación internacional, que restringe las alternativas estatales (Neves, 2001). Y la referencia persistente es a la necesidad de castigo penal para los guerrilleros por crímenes de lesa humanidad. El discurso económico se dice más flexible, pero en el entendido de que la economía global tampoco permite cambios soberanos significativos en el contexto nacional y esta supeditada a políticas de mediano y largo plazo en proceso. Allí predomina la retórica de la disminución de la desigualdad en la distribución de los bienes, fundamentalmente a través del crecimiento económico. Y en el discurso social, hay una tendencia a atribuir la mayoría de las perturbaciones y desordenes, a la existencia de los grupos guerrilleros. Es decir, a las cualidades morales e intelectuales de un grupo de personas, que por razones incomprensibles, subvierten el orden presupuestamente bueno de la sociedad.

Entre los discursos gubernamentales esbozados, el discurso jurídico ostenta la mayor coherencia y estabilidad, no obstante cuestionada por conatos de corrupción e infiltración de sectores sociales adversos al proceso de paz, tanto de la izquierda como de la derecha. Pero además, destacan unilateralmente el carácter ejecutivo del ordenamiento jurídico, ocultando o invisibilizando el proceso legislativo y doctrinario, en donde hay lugar para la revisión de las normas de convivencia, la interpretación del bien común y la concertación de normas alternas de participación política (Neves, 2001).

En cuanto al discurso económico, a pesar de la aparente flexibilidad e interés por la disminución de las desigualdades en la distribución de la riqueza, es palpable el apego al modelo de economía de mercado, cuyas leyes se consideran naturales e inamovibles. La fuerte influencia de los grupos económicos es interpretada por los medios masivos de comunicación, que descalifican permanentemente las observaciones alternas como inviables, producto de la ignorancia o la candidez. Y fenómenos de mercado como el tráfico de drogas, el contrabando y los delitos contra la propiedad, se atribuyen exclusivamente a la criminalidad y a la inmoralidad de los grupos alzados en armas.

Finalmente, en cuanto al discurso social, abiertamente se señala a los guerrilleros como victimarios primitivos y al pueblo (del cual parecen excluirse los dirigentes políticos y los estratos altos), como la víctima, de una guerra, que se da a entender, puede proseguirse indefinidamente. En esta dimensión, la guerra se presenta como un espectaculo para las élites, que se asombran ante la barbarie de sus participantes. Incluida la pauperización, la marginalización y la exclusión de las víctimas, que si no se le atribuye directamente a los guerrilleros, se le adjudica a la insolvencia moral, a la incapacidad o a la inferioridad de sus victimarios.

\section{La sociedad de la guerrilla}

El discurso de la guerrilla respecto de la sociedad que pretenden, es poco difundido y sólo conocido por algunos expertos de la academia y de los sectores políticos en contienda. Trasciende principalmente el tema agrario, el tema de la injusticia social, el tema de la participación política y el cuestionamiento a las instituciones del Estado, por su corrupción, ineficiencia y falta de soberanía. Sin embargo, aparte de la exigencia de participación política y democratización del Estado, no se conocen las concepciones de sociedad que imaginan. Y por contraste, la actividad económica a la que se les vincula (tráfico de armas y de estupefacientes, extorsión y secuestro), parece indicar que se han adaptado muy bien a las reglas de una economía de mercado. 
Paralelamente, en el nivel de la semántica, la izquierda y la guerrilla se asocian persistentemente y los activistas de derechos humanos, de los movimientos sociales y las re-invindicaciones civiles, se ubican como socios estratégicos del proyecto subversivo, al que le corresponde, por el Estado y sus mecanismos de inteligencia, una estrategia contra-insurgente consistente en la descalificación sistemática del programa de la izquierda política.

\section{La sociedad realmente existente y las expectativas frente a los diálogos de La Habana}

Frente a estas descripciones de la sociedad de las partes en diálogo en La Habana, conviene hacer referencia a las otras descripciones alternas y a las expectativas consiguientes, de los múltiples sectores sociales que conforman nuestra nación. Primero hay que hacer alusión a las distinciones que configuran nuestro mapa poblacional: citadinos y campesinos, ejecutivos y obreros, trabajadores formales e informales, comerciantes formales e informales, hombres y mujeres, jóvenes y ancianos; atravesados y divididos por su arraigo local y su exposición a una cultura global propiciada por las nuevas Tecnologías de la Información y la Comunicación.

Se ha convertido en principio de la convivencia cotidiana la tan mentada glocalidad: "pensar globalmente y actuar localmente". La extensa exposición de toda la población a los mass media, incluidas las redes sociales y el internet, permiten suponer que los contenidos de la consciencia, aunque diversos, están circunscritos mayoritariamente a la cultura Hollywoodense, en segunda medida a la cultura Europea y por último a nuestros vecinos más influyentes (Argentina, Brasil y Chile). Es decir, la semántica de la sociedad está atravesada por el predominio de la cultura espectáculo y del ciudadano turista, que nos posicionan como transeúntes de nuestro territorio, ávidos de novedades en el primer mundo y decepcionados de nuestras condiciones de existencia. No obstante, implicados, enredados, angustiados, estresados por las rutinas diarias, cada vez más pautadas por una monetarización extendida de la convivencia, que deteriora la confianza en nuestros congéneres y aumenta la incertidumbre y el riesgo (Luhmann, Familiarity, Confidence, Trust: Problems and Alternatives, 2000), estamos cada vez más integrados por descripciones de la sociedad de tipo religioso, en el que la salvación individual se convierte en la estrategia más frecuente. Y en la que prevalecen las distinciones estéticas de lo bello y lo feo asociados a lo bueno y lo malo, respectivamente, como es propio de las sociedades estratificadas; en vez de la diferencia más funcional entre lo interesante y lo aburrido (Schmidt, 1999).

\section{El conflicto y su naturaleza constituyente de lo social}

El que el conflicto sea considerado un elemento perturbador del orden social, corresponde a la tradición moderna dominante según la cual lo social se concibe como contrato, consenso o acuerdo (Luhmann, Complejidad y Modernidad. De la Unidad a la Diferencia, 1998). Y a la socialización correspondiente como indoctrinación, sometimiento, disciplinamiento, dominación, y/o violencia simbólica. (Foucault, Vigilar y Castigar, 1986). Una descripción alternativa de lo social como operación de comunicación autopoiética, cuyo origen sea la contingencia de la relación Alter - Ego, su recurso, la reducción de complejidad para la consciencia y el resultado, la reiterada posibilidad de disentir, como condición para generar estabilidad (Luhmann, Sistemas Sociales: Lineamientos para una teoría general, 1992), justifica un desarrollo más elaborado.

\section{La contingencia de lo social: la relación constitutiva Alter - Ego}

Con la ayuda del siguiente esquema, se puede esclarecer la distinción social frente a lo psíquico y describir la operación de comunicación constitutiva de lo social. 
Es premisa de la descripción de la sociedad proporcionada por Niklas Luhmann (Luhmann, Sistemas Sociales: Lineamientos para una teoría general, 1992), que las operaciones de la consciencia constituyen el entorno para las operaciones de comunicación, cuya producción esencial es la comprensión resultante de la percepción que hace Ego de las afirmaciones de Alter con respecto a una determinada selección de información (Schmidt, 1999). Para el caso, el tema del diálogo entre el gobierno y la guerrilla de las FARC en La Habana, es empleado por cada ciudadano (Alter) para definir su postura frente a otro (Ego), que ve en esa disposición sus opciones de cooperar o disentir. Es importante subrayar acá, que la información que es objeto de selección en este caso, es la relativa a las declaraciones (es decir percepciones) que tiene cada uno de las partes en la mesa de La Habana, como se definen a sí mismas en el proceso. Y por ende, constituye información elaborada por los medios, que no es empleada directamente en el quehacer cotidiano, sino de la que se dispone como insumo de reserva, para discriminar, de manera gruesa, entre enemigos y amigos. Y esta percepción entre amigos/enemigos es la que sirve como selección cotidiana para que los actores sociales tomen sus decisiones en las operaciones de comunicación social.

Antes de decidir la justeza, prudencia o pertinencia de dicha distinción (amigo/ enemigo), lo que cabe es subrayar que ese no es el único lazo social posible. Más aún, habría que decir que dicho lazo social fundado en valores comunitarios (Luhmann, Familiarity, Confidence, Trust: Problems and Alternatives, 2000), se corresponde mejor con una sociedad estratificada integrada verticalmente, proclive a la violencia simbólica de la sobre-integración

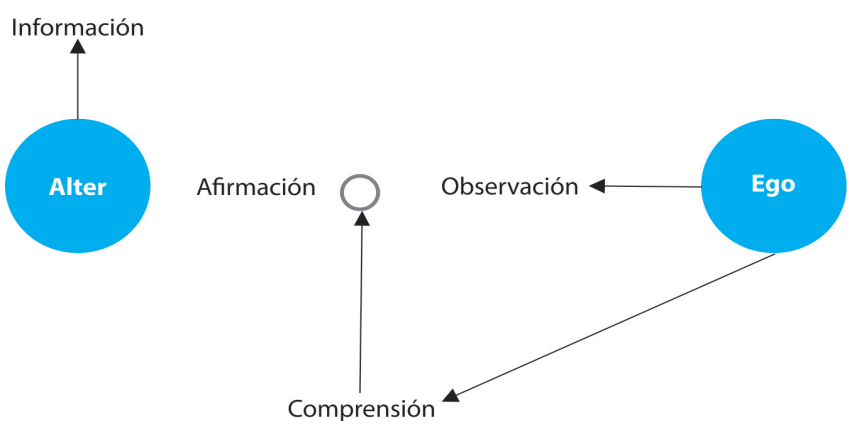
y la sub-integración social (Neves, 2001)².

\section{La participación: reducción de complejidad para la consciencia}

Como se indicó anteriormente, uno de los efectos psicológicos de la inoperancia de una descripción efectiva de la sociedad, que le dé sentido a la acción social, es el aumento de la incertidumbre y su absorción mayoritaria por el discurso religioso. Discurso que en los tiempos modernos estimula la desconfianza hacia el semejante, manifiesta como síntoma en los fenómenos de acoso y xenofobia, promoviendo la confianza en Otro abstracto (Dios). Y absorbiendo el alto riesgo inherente a las sociedades funcionales (contingentes), mediante prácticas rituales que acaparan las opciones de sentido.

La tendencia de la sociedad del conocimiento, es a equiparar el empoderamiento con una representación apropiada de la realidad. Sólo quien sabe suficientemente, puede actuar eficientemente. Y, al nivel de la semántica de la sociedad, la integración social depende fundamentalmente del sistema educativo, que da acceso al sistema económico; mientras que el sistema político, es de la esfera de interés de las élites. Mientras que al nivel de la praxis social, son las élites económicas las que toman las decisiones políticas, que determinan las posibilidades de inclusión social. Al punto que, al sistema jurídico se le atribuyen por parte de los movimientos sociales, la criminalización de la protesta social.

2 "The overintegration pole has access to the products and benefits of the social systems, without being simultaneously dependent on their constraints and rules (positive integration), while the under-integrated pole is dependent on the rules and constraints without having access to the products and benefits (negative integration), so that at both poles, defective inclusion as well as partial exclusion exists." (Neves, 2001). "El polo sobreintegrado tiene acceso a los productos y beneficios de los sistemas sociales, sin ser simultáneamente dependiente de los constreńimientos y normas (integración positiva), mientras que el polo subintegrado es dependiente de las normas y constreńimientos, sin tener acceso a los productos y beneficios (integración negativa), de tal forma que en ambos polos, existe tanto la inclusión defectuosa como la inclusión parcial”. 
En este panorama, lo que interesa destacar es la contradicción persistente entre los esfuerzos de los medios de comunicación al insistir en una semántica de lo bello / feo, apoyada en las campañas publicitarias de las multinacionales, que se sustentan en la proliferación de la belleza corporal (todavía siguen vigentes los reinados de belleza), propia de una sociedad estratificada; y la necesidad de una sociedad organizada funcionalmente, de promover el interés de sus ciudadanos por involucrarse en actividades sociales específicas, evitando el aburrimiento propio de la exclusión y la marginalidad.

\section{La dinámica de estabilización / desestabilización del conflicto}

En el entendido de que la contingencia de las relaciones sociales es inherente a la constitución de lo social y de que los actuales niveles de exclusión son síntoma de una sobre-integración / sub-integración, derivada de una semántica inapropiada para una sociedad funcionalmente organizada, se comprende por qué el papel del conflicto varía entre una sociedad representada utópicamente como proyecto y la sociedad actualizada permanentemente como comunicación (acciones coordinadas basadas en la comprensión de su finalidad).

No hay duda de que en la semántica social, hoy en Colombia prevalece la idea de sociedad armónica, pacífica, integrada espontáneamente, en la que el conflicto es un ruido que la desestabiliza indeseablemente. Y por eso la añoranza de la sociedad post-conflicto. Pero tal representación no soporta un mínimo acercamiento a la realidad, donde los conflictos políticos, económicos y sociales pululan por doquier; y aunque se sigan etiquetando como perturbaciones indeseables, insisten de tal forma que más vale revisar su papel.

Vistas las cosas desde la perspectiva de la variedad de percepciones realmente existentes, como posiblemente antagónicas, la estabilidad social no puede buscarse por el lado del consenso y la armonía abstractas; sino más bien, por el lado de promover construcciones alternativas de la realidad, que producto de los intereses ciudadanos. Y eso supone, primero, descartar la posibilidad de que las personas, a título individual, se puedan hacer a una representación suficiente y adecuada de la realidad, que por suficiente y adecuada, coincida con la de las otras personas, evitando así el conflicto. Más bien lo contrario es cierto. Las representaciones del mundo que cada persona se hace, son en cada caso particulares y relativas al horizonte de su experiencia. Y dichas representaciones están al servicio de comprenderse con aquellos a los que se asocia, con funciones también particulares y específicas. No hay cosmovisiones, ni correlatos universalmente válidos; sino solo actuaciones pertinentes, cuyo sentido siempre es susceptible de discutirse. Segundo, que exista una representación de sociedad con respecto a la cual se puedan proyectar los esfuerzos colectivos para conseguirla, aun a costa de sacrificar el presente de nuestra actividad (p.e. la guerra). Sino que lo que existe, es una actividad social permanente, que animada por diversos sentidos, se encuentra en permanente conflicto, hallando en ocasiones diversos grados de estabilidad.

Así, el conflicto no es un elemento accesorio, al que habría que eliminar, sino un ingrediente esencial en la constitución de lo social, que tendría que animarse para estimular la consecución de operaciones sociales estables (ecológicamente perdurables y sostenibles).

\section{La participación social y la democracia}

Si a una sociedad estratificada le corresponde el gobierno autorizado de una élite ilustrada, en la que los subalternos tienen todo por hacer y nada que decir (Spivak, 1988); a una sociedad funcional le corresponde el gobierno de sí misma por un mecanismo de participación democrática, en el que todos los sectores sociales comprenden su interdependencia y se interesan cada vez más por atender 
los problemas que surgen de su respectiva actividad (Arendt, 1993). La gestión del conflicto y no su eliminación, porque su eliminación es equivalente a la sepultura de la actividad social, como lo demuestran experiencias tan recientes como los campos de concentración Nazi en la primera mitad del siglo XX. Y los recurrentes genocidios del que los medios de comunicación nos informan, que son el resultado de conflictos armados.

Con la premisa de que la comunicación social (la actividad social cuyo sentido se entiende) es la que promueve el interés de las consciencias a involucrarse, y viceversa, las consciencias altamente interesadas en las funciones sociales son las que contribuyen a coordinar acciones con sentido; el conflicto armado iría desapareciendo como un esfuerzo estéril de imponer a otro un proyecto social único (universal), para dar paso al conflicto social como operación permanente de reproducción de sentido diverso e implicativo. Esta organizació de lo social por el interés diverso de sus actores, supone la responsabilidad colectiva en la producción de información y la generación de expectativas de expectativa de conflicto, y no de armonía social.

\section{La dimensión objetiva: la información}

En lo relativo a la información, cuya producción y circulación corre a cargo casi que exclusivo de los mass media, y que constituye la referencia de selecciones posibles para la comunicación social; se pueden diferenciar los tipos predominantes de acuerdo a las franjas de interés: informativa con los noticieros, que incluyen política, deporte, farándula y sociales; entretenimiento con seriados, dramatizados, novelas y concursos; y educativa.

La información noticiosa incentiva la participación contemplativa del ciudadano, mientras que el entretenimiento alimenta sus imaginarios de lo ideal y la información educativa mantiene su dependencia de los expertos (Rey, 2000). La familiaridad o el mundo común que se produce por esta vía, promueve la subintegración de las mayorías expuestas a la cultura de masas y la sobreintegración de las minorías cultivadas para la administración y la dirigencia. Lo que ha promovido un divorcio entre las prestaciones y la reflexión, o entre la operación y la planeación, entre el hacer y el saber. La consecuencia es que gran parte de la actividad social se ha automatizado y no tiene sentido para quienes la realizan. Y entonces, el sentido se desplaza hacia lo imaginario individual o marginal.

Una manera de revertir esta tendencia, es involucrando a los actores sociales en el sentido de sus actividades cotidianas, promoviendo la reflexión sobre su quehacer y la toma de decisiones para replantear las situaciones sociales, recreando las distinciones posibles (esto es multiplicando la generación de información prácticamente relevante). El fenómeno de las redes sociales puede ser indicador del potencial en esta dirección.

\section{La dimensión temporal: la expectativa y las expectativas de expectativa}

Dado que las operaciones de comunicación como las de consciencia son eventos necesarios a la estabilidad de la sociedad en un caso y a la identidad de la persona en el otro, requieren del tiempo como mecanismo que difiere sus relaciones mediante la producción de expectativas. La consciencia opera con base en expectativas, que van desde la absoluta certeza (el ciclo solar, el ciclo vital, las leyes de la física, por citar algunas) a la incertidumbre asumida como riesgo (p.e. escoger una profesión, elegir un automóvil, comprar o arrendar una vivienda, contraer matrimonio, comprar un billete de lotería, invertir en un negocio). Y estas expectativas de la consciencia, se conectan con las expectativas de expectativa. Por ejemplo, al elegir una profesión, se tienen en cuenta, además de las opciones personales para desarrollarla, la remuneración esperada, el estatus social que supone, la estabilidad que genera; 
todo lo cual constituyen expectativas de la expectativa de los otros. Esto corresponde a la noción de confianza, definida como la mayor estabilidad de las expectativas de expectativa (Luhmann, Familiarity, Confidence, Trust: Problems and Alternatives, 2000), lo cual garantiza la planificación a mediano y largo plazo y se traduce en la experiencia de seguridad.

Cabe preguntarse si, como pretende sugerir el discurso gubernamental de los diálogos con la guerrilla de las FARC, los inamovibles (el marco jurídico y el modelo económico) se traducen en la seguridad prometida y anhelada por los ciudadanos. Lo primero que cabe observar, es que la confianza derivada de la autoridad de quien declara un estado de cosas, es la confianza propia de una sociedad estratificada, pre-moderna, que en últimas apela a la autoridad de Dios, para anular las inquietudes que de todas formas surgen entre los ciudadanos, que puede dudar de la suficiente ilustración de sus autoridades.

La confianza, en una sociedad funcional, en la que la multiplicidad y diversidad de información disponibles, hace imposible la autoridad personal, se funda más bien en la capacidad y experticia de los subsistemas sociales (p.e. educación, salud, economía, política) para absorber funcionalmente los problemas correspondientes y realizar las prestaciones sociales necesarias a la estabilidad de la organización. Por lo que se ve, el proceso de diálogos en La Habana se funda más bien en la autoridad de las declaraciones, que en la pericia de los diferentes subsistemas para suministrar una adecuada descripción societal de las dimensiones económica, política y social.

\section{La gestión social del conflicto}

Llegados a este punto, hay coincidencias actualmente en que las sociedades dependen fundamentalmente de la planificación experta. Quizás la discrepancia radica en a quien se le atribuye esta experticia. Por un lado, tanto las corrientes neo-liberales como las socialdemócratas, tienden a atribuírselo a personas especialmente calificadas tanto moral como intelectualmente. Mientras que desde una perspectiva menos humanista y más societal (Badiou, 1999), se le atribuye a subsistemas sociales especializados, que generan en sí mismos información específica y dictámenes independientes de intenciones programáticas (Molbak, 2012).

\section{Los temas de conversación}

Por tanto, no se trata de uniformar el universo simbólico, sino más bien de diversificar los códigos de comunicación social, permitiendo la emergencia de nuevos tópicos de conversación cotidiana, que convocan el interés de los ciudadanos de múltiples y diversas maneras. De ahí que lo malo de nuestra sociedad actual, no es tanto la monopolización de la riqueza, como la monopolización del discurso y de las ideas. La concentración del conocimiento que se corresponde con la concentración del capital, es lo que verdaderamente arriesga la estabilidad social.

\section{Las expectativas de expectativa}

Por consiguiente, las expectativas de armonía social resultado del consenso, son el resultado deuna imposición anacrónica de una representación de la sociedad estratificada e integrada moralmente. Mientras que la operación social se anima permanentemente por el conflicto que surge de la libertad de discrepar con visiones alternas. Una sociedad funcionalmente integrada, requiere del interés de los agentes sociales por vincularse a sus rutinas especializadas y no de la anomia de trabajadores automatizados (Dijk, 1999). 


\section{Conclusión}

Una de las consecuencias del proceso de paz encaminado a dirimir el conflicto armado en Colombia, ha conducido a la representación de la utopia de la sociedad como armónica y fundada en el consenso ilustrado de sus dirigentes. Un análisis más cercano de la situación, revela representaciones alternas de la sociedad, que demandan una participación democrática, dinamizada por el conflicto que se suscita por la diversidad de percepciones y la multiplicidad de funciones a las que nos vemos abocados en el mundo contemporáneo.

\section{Referencias}

Arendt, H. (1993). La condición humana. Barcelona: Paidos.

Badiou, A. (1999). El Ser y el Acontecimiento. Madrid: Manantial.

Calviño, M. (2002). Ser Psicóloga Hoy (y mañana...). Revista Cubana de Psicología, 3-8.

Dijk, T. B. (1999). A theoretical frame of reference for family systems therapy? An introduction to Luhmann's theory of social systems. Journal of Family Therapy, 195-216.

Foucault, M. (1986). Vigilar y Castigar. Madrid: Siglo XXI.

Luhmann, N. (1992). Sistemas Sociales: Lineamientos para una teoria general. Mexico: FCE.

Luhmann, N. (1996). The Reality of the Mass Media. California: Stanford University Press.

Luhmann, N. (1998). Complejidad y Modernidad. De la Unidad a la Diferencia. Madrid: Editorial Trotta.

Luhmann, N. (2000). Familiarity, Confidence, Trust: Problems and Alternatives. En D. Gambetta, Trust: Making and Breaking cooperative relations (págs. 94-107). Oxford: University of Oxford.

Molbak, R. L. (2012). From a Phenomenology of the Subject to a Phenomelogy of the Event: Reconstructing the Ontological Basis for a Phenomenological Psychology. Journal of Phenomenological Psychology, 43(2), 185-215.

Neves, M. (Junio de 2001). From the Autopoiesis to the Allopoiesis of Law. Journal of Law and Society, 28(2), págs. $242-264$.

Ort, N. (1999). Systems Theory with Lacan. Germanic Review, 74, 30.

Rey, F. G. (2000). El lugar de las emociones en la constitución social de lo psíquico: El aporte de Vygotski. Educación y Sociedad, 132-148.

Schmidt, B. M. (1999). Systems Theory and Literary Studies in the 1990s. Germanic Review, 74, 242.

Spivak, G. C. (1988). Can the Subaltern Speak? En C. N. Grossberg, Marxism and the Interpretation of Culture (págs. 271-313). Chicago: University of Illinois Press. 